\title{
El impacto de la globalización sobre la gestión estatal en economías no desarrolladas
}

\section{Maribel Barboza Pérez*}

\section{Resumen}

Se discute la Globalización como un proceso de integración/exclusión de los flujos económicos autonomizados, en el macrosistema económico, que lesiona las claves regulatorias tradicionales asociadas a la entidad Estado-Nación. Las economías inmaduras son receptoras de los efectos desestructurantes de tal "anarquía liberalizadora", al carecer de soportes protectivos. Ya vulneradas por sus ineficiencias internas, por la cesión negociadora de soberanía, implicita en los programas de ajuste, y las dificultades integrativas, han de exponerse, en ausencia de estrategias nacionales, a un liberalismo asimétri$\mathrm{co}$, en búsqueda de las ganancias que el "mercado amigable" washingtoniano les asegura. A pesar de los resultados asimétricos, expresados en la concentración de los beneficios y flujos del comercio en las economías del "primer mundo", el acceso exitoso de las Economías de Industrialización Reciente a los circuitos de la globalización se erige como referente para la construcción de estrategias de crecimiento sustentadas en "nuevos" supuestos: la intervención pública "desarrollista" como generadora de ventajas competitivas selectivas.

Palabras clave: Globalización, selectividad, Estado desarrollista, comercio internacional, intervención estatal.

Recibido: 21-10-97 . Aceptado: 10-11-97

* Economista, Investigadora del Instituto de Investigaciones Económicas, Facultad de Ciencias Económicas y Sociales, Universidad del Zulia. E-mail: marecon@ven.net. 


\title{
The Impact of Globalization on State Action in Non-developed Economies
}

\begin{abstract}
This paper discusses globalization as a process of integration/exclusion from autonomous economic action in the economic macrosystem, which interrupts the traditional regulatory controls of nation-states. The immature economies receive the de-structuralizing effects of this "liberalizing anarchy" and have no protective supports in place to defend themselves. Already weak due to internal inefficiencies, and by the loss of economic sovereignty implied through the adjustment programs and difficulties of integration, they are exposed,in the absence of national strategies, to an asymmetric liberalism, in the search for income from the "friendly market" that Washington assures them. In spite of the asymmetric results, expressed in the concentration of benefits and economic flows in first world economies, the successful access of recently industrialized economies in the globalizing circuits arises as a reference point in the construction of strategies of sustainable growth in new proposals: public development intervention as a generator of selective competetive advantages.
\end{abstract}

Key words: Globalization, Selectivity, Development state, International commerce, State intervention.

\section{La globalización: tendencia de inclusión-exclusión}

La globalización es un fenómeno aún sin definición acabada; Hirst $y$ Thompson (1992) ${ }^{1}$ por ejemplo, cuestionan su carácter de "factum" proponiendo ésta como una tendencia, por demás reversible. L. Bendesky $(1994)^{2}$ asume la misma posición sobre el relativismo globalizador enjuiciando la tesis de "mundo sin fronteras" o "fin de las geografias". Una tesis opuesta es la de "extrema globalización" de K. Ohmae (1991), para quien la mundualización constituye el nuevo orden mundial, sin posibilidad regresiva.

1 P. Hisrt y $\mathrm{H}$. Thompson (1992), desafian el concepto de globalización como "ideal type" descriptivo de un nuevo orden mundial; argumentan que este concepto más bien es alusivo a "tendencias que pueden involucionar o detenerse de acuerdo a la dinámica de la economia". Proponen, en sustitución, el concepto de economía internacional.

2 L. Bendesky (1994) cuestiona la "escala planetaria" que se ha asignado al proceso globalizador: "Sólo describe algunas de las formas de operación de las grandes empresas y de ciertos mercados alrededor del mundo". 
Tal divergencia desaparece sin embargo, al abordarse las manifestaciones e incidencias asociadas al fenómeno estudiado $y$, en tales términos, hemos construido una definición aproximativa: se entiende como globalización al proceso de integración de mercados y naciones a través de flujos autonomizados financieros, de bienes y servicios, soportados en tecnología productiva y comunicacional, caracterizado por su carácter simultáneamente excluyente y diferenciador y con claves regulatorias lesivas a la entidad Estado-Nación. De acuerdo a L.Bendesky (1991) ha sido, precisamente, la generación simultánea de cooperación y conflicto, y el impacto de extrema desigualdad sobre distintas economias lo que configura "uno de los límites a la paradoja de la propia globalización".

La concreción de este proceso podría encontrarse en la exacerbación de ciertas tendencias estructurales del comercio internacional evidenciadas a partir de los años ochenta: más alta concentración del mismo entre las economias desarrolladas y algunas de las economías de industrialización reciente (EIF), crecimiento del comercio intra-industrial entre éstas, así como la tasa de emisión/recepción de los flujos de inversión ${ }^{3}$. Estos indicadores verifican el registro de la segregación en un mundo "integrado" (interlinked economy) $^{4}$ conformado por bloques y proto-bloques diferenciables (NAFTA, UE y Japón) y un mundo "desintegrado" constituido por los PND.

En este nuevo orden, las economías "integradas" (Asociación de Libre Comercio de América del Norte -NAFTA, Unidad Europea -UE- y Japón) y de alto potencial integrativo (EIR y emergentes) pueden, y de hecho asi ha sido, generar conductas reactivas de carácter concéntrico en torno a ejes regulativos supranacionales. Al margen de los mismos existen áreas grises de países no desarrollados rezagados de la dinámica de crecimiento mundial. Estos reciben doblemente el impacto negativo de la globalización: su capacidad de manejo macro/microeconómico está sujeto, no sólo, a los impulsos del comercio internacional determinados por el mundo "integrado", sino también, a las "conditionalities" establecidas en la programación concertada con los organismos multilaterales. La vulnerabilidad implicita restringe tanto la posibilidad de construcción de una "estrategia nacional de crecimiento" como el éxito de las asociaciones defensivas regionales.

Puede decirse entonces, que la globalización intensifica la dualización

3 Pueden encontrarse en J.Markusen (1995) indicadores sobre la concentración del comercio internacional: a) los paises desarrollados son los mayores emisores/receptores de inversiones extranjeras, en la última década emitian el $97 \%$ de la inversión directa y recibian el $75 \%$ de la misma. b) los flujos de inversión generados por miembros del G-5 son absorbidos en un $70 \%$ por sus pares (prevalencia del principio del similaridad). c) El crecimiento del comercio intra-firma ocupó el $30 \%$ del comercio mundial.

4 P. Hist y $H$. Thompson estructuran el sistema económico mundial en los siguientes bloques, proto-bloques, y economías: NAFTA/USA, UEJEFTA, JAPON \& Nics, ex-USSR, Países no desarrollados (PND), Caim group + Argentina, China y Europa del Este. 
del orden económico mundial ${ }^{5}$; en otros términos, la "systematic interdependence" que la define supone, sincrónicamente, "formas desiguales de interdependencia" (Bendesky, L. 1994).

\section{Las nuevas claves regulatorias}

La economía globalizada lleva consigo tendencias que fracturaran el orden económico de la post-guerra, "la Pax Americana" (Hirst, P. y Thompson, G. 1992). La apertura y desregulación contribuyen, irónicamente, a la fragmentación de la hegemonía norteamericana dando lugar a un sistema de hegemonias, compartidas 0 , en términos de $F$. Sagasti y G. Arévalo (1992), "...Estados Unidos empieza a ejercer lo que podria llamarse una "hegemonía diluida"..." EI nuevo orden invalida las claves regulatorias precedentes impactando, en diversos grados, las instituciones económicas y políticas que les sustentaban: Bretton Woods-FMI (regulación monetaria financiera internacional); Estado de bienestar (regulación y legitimidad interna); Acuerdo General sobre Aranceles Aduaneros y Comercio -GATT- ahora Organización Mundial del Comercio-OMC- (regulación comercial), ONU (regulación política internacional) y Estado-Nación (regulación política nacional). Analicemos cómo ha sido el proceso de invalidación de las claves referidas:

\section{1) Regulación financiera}

La internacionalización de los mercados financieros se hace posible, gracias a la desregulación de los mismos impulsada por eventos tales como la instauración del régimen de libre flotación y al exceso de liquidez (fondos petroleros) atrapado en el circuito financiero internacional durante los años setenta. Si bien esto amplifica y le confiere autonomía a los flujos financieros, la volatilidad inicia la caracterización del sistema financiero internacional; el problema de la deuda y los sostenidos imbalances externos de la economía estadounidense signan su interrupción. La respuesta a la desestabilización financiera fue la "regulación, ya no dirigida univocamente por políticas de FMI, sino también por entidades regulatorias supranacionales tales como el G-8 y la UE (Maastricht) a través de monitoreos monetarios permanentes ${ }^{6}$.

La misma incontinencia financiera, obviamente, encuentra débiles respuestas regulatorias en el ámbito estrictamente nacional. Ha sido precisamente, un rasgo asociado a la globalización, la pérdida de efectividad regulatoria estatal de las políticas monetarias domésticas; de

5 El fenómeno de la dualización es expresado por F. Sagasti y G. Arévalo (1992) como "orden global fracturado" en referencia a la co-existencia de procesos de globalización con rupturas entre países, regiones y grupos sociales.

6 La efectividad de la regulación supranacional, específicamente del Sistema Monetario Europeo, pudo comprobarse con el control de la crisis especulativa del verano de 1993. 
alli la necesidad agregativa. Ahora bien, si aún las mismas economías integradas son sensibles ante la autonomía transactiva financiera, es imaginable la vulnerabilidad externa de los países no desarrollados y los grados de impotencia de sus politicas monetarias internas. En éstos, las claves regulatorias monetarias adicionan a sus propias incompetencias internas, las demandas, paradójicamente desregulativas, impuestas en los programas de ajuste. Las mismas han generado, por ejemplo, en el escenario latinoamericano, crisis financieras iterativas; así como la evidencia de la pérdida de autonomia financiera-monetaria de los gobiernos nacionales.

\section{2) Regulación comercial}

El Gatt-OMC, conjuntamente con la Conferencia de las Naciones Unidas sobre Comercio y Desarrollo -UNCTADson reconocidas como las instituciones claves que, nominalmente "regulan" el comercio internacional. Esta estructura normativa, sin embargo, ha sido lesionada por la globalización: se ha develado su disparidad discursiva-operativa y su capacidad normativa se ha marginalizado ante la agresividad de los esquemas endoregulatorios de los bloques integrativos regionales, especificamente, U.E. y NAFTA.
Es destacable que, mientras la institucionalidad del "primer mundo" se sustenta en una doctrina del comercio que promueve la liberalización comercial progresiva, induciendo a la eliminación de "impedimentos estructurales al acceso del mercado o de deformaciones al comercio" (M. Agosin y D. Tussie, 1993), refiriéndose a los principios de reciprocidad, neutralidad y transparencia, la estructura del comercio mundial sugiere movimientos y prácticas abiertamente discriminantes. Estos movimientos se descifran en la tendencia a la convergencia, cuya expresión más acabada la constituyen los bloques de comercio. Estas nuevas locaciones regulativas generan normas de conducta (proteccionistas) inter e intralocus al margen de la institucionalidad reconocida. De alli que sea identificable un neo-proteccionismo abiertamente exclusor de economías no integradas.

Esta especie de "liberalismo negociado" (M. Agosin y D. Tussie (1993)) reedita prácticas configurativas de restricciones de acceso de países no desarrollados al circuito del comercio mundial; lo que colide no sólo con la incitación a la apertura y liberalización comercial inscrita en los programas de asistencia de los organismos multilaterales, por ejemplo, los Structural Adjustment Loan SAL (Préstamo para ajuste estructural) del Banco Mundial ${ }^{7}$, sino también las estrate-

7 Los programas del BM orientados al crecimiento en el mediano plazo son conocidos como SAP (structural adjustment programmes) que permiten el acceso a dos tipos de préstamos: SECAL y mini-SECAL (sectorial adjustment loan). Un análisis exhaustivo de la naturaleza y restricciones de estos programas puede encontrarse en J.B. Opschoor y J.M. Jongma (1996). Una literatura adicional sobre este "issue" se encuentra en D. Greenway y O. Morrisey (1993). 
gias de ajuste "estandarizadas" hacia la industrialización orientada a las exportaciones -EOI- de Estado pasivo, establecidas como la única opción disponible de crecimiento para los países no desarrollados.

De forma tal que, a pesar de un publicitado "friendly market" los países desarrollados no reciben tratamiento de "socios de pleno derecho" y han de lanzarse a una exhaustiva apertura sin reciprocidad y en ausencia de estrategias protectivas públicas.

\section{3) Regulación Estado-Nación}

Resulta verificable el que la globalización lesiona, o cuando menos altera, el concepto tradicional de Estado-Nación asi como su expresión gubernamental de corte welfarista. El soporte argumental de la prevalencia del mercado eficiente $e$ irrestricto, conduce, de acuerdo a Hobsbawm (1996), a una suerte de "anarquismo ultraliberizador" en el cual se tiende al desmontaje de las claves nacionales (identidad-Estado-gobiemo); estas podrian erigirse, de acuerdo al paradigma liberal, en interferentes en la construcción de los nuevos "locus" transnacionales en la "relocalización" de las actividades productivas y financieras en el nuevo espacio global. A partir de este "desideratum" se ha configurado el "New Ideal" 8 , pro- puesta que establece el retorno al Estado gendarme o "minimal state".

El Estado inicia entonces un proceso de subsidiarización con respecto a las entidades erigidas en nuevas claves regulatorias: las instituciones supranacionales y las compañías trasnacionales -TNC-, a tal propósito denominadas "stateless corporation" (Hirst P. y Thompson, H. 1992). Su acción transnacional evidencia, no sólo la îrrelevancia de los territorios nacionales, sino también la pérdida de autonomia de los Estados sobre las actividades económicas que en éstos se registran. En este sentido se ha llegado a definir al Estado como "la autoridad local del sistema global" (Hirst P. y Thompson, G. 1995); esta aceptación confina al mismo a una suerte de autonomia "inferior".

Aún cuando se disiente sobre los grados y fuerza anulatoria que operan sobre la soberanía, sí existe consenso en cuanto a que ésta, por lo menos, altera su sentido institucional tradicional. E. Hobsbawm (1996) aduce, al respecto, que la soberanía estatal se "ve reducida ante la fuerza de entidades supranacionales", así también, la "capacidad de dirigir sus propias economias". P. Hirst y H. Thompson (1992) refieren la "declinación de los distintos poderes disciplinantes de los Estados aún cuando la ciudadanía permanezca en los límites nacionales". D. Ibarra (1993) encuentra en el debilita-

8 El "new ideal" define la "nueva" tesis liberal sobre el Estado (minimalista), a partir de la cual se devuelve al mercado y los agentes privados la decisión sobre la asignación de los recursos. Puede leerse en M. Bruno (1994). 
miento del Estado "el correlato sistemático" de la globalización; asi también sugiere que, en tal contexto, éste ha de renunciar al "uso de un buen número de instrumentos del viejo arsenal de políticas nacionales". En suma, son profusas las reflexiones que establecen la incidencia relativa de la transnacionalización económica sobre el ejercicio de autonomía estatal, en el manejo macroeconómico doméstico.

\section{Impacto asimétrico de la globalización}

Asumiendo la validez de la hipótesis anterior, se hace necesario preguntarse si este impacto es simétrico para la economía global. La respuesta es, nuevamente negativa. La magnitud del impacto dependerá de la vulnerabilidad de los Estados y ésta responde a la "gobernabilidad como a la contestabilidad" lograda por los mismos (E. Ortiz 1996).

En el "primer mundo" los paises han logrado, no sólo, construir instituciones cooperativas-regulativas supra-na- cionales, sino que además, a pesar del signo federativo, preservan sus identidades nacionales. De forma tal que han consolidado fómulas de gobernabilidad intra e inter-Estados que les permiten desarroliar estrategias reactivas de defensa y agresión frente a los efectos adversos de la globalización ${ }^{9}$.

Los paises no desarrollados, al ser presionados a una apertura indiscrimina$\mathrm{da}$, en ausencia de los dos soportes mencionados, presentan una reducción significativa de su soberanía. ¿Por qué se someten a esta tendencia de subsidiarización?. Su expediente de estrategias económicas desacertadas, por lo general autoexclusivas (proyectos políticos "nacionalistas" y estrategias económicas endo-orientadas) ${ }^{10}$, les han conducido a una desestabilización sistemática y de allí a la adopción, en plazos diversos, de programas estandarizados de estabilización y ajuste como condición de acceso a la asistencia financiera de los organismos multilaterales. La recurrencia a los mismos implica la ordenación univalente de las economías receptoras bajo un mode-

9 Se ha señalado que las economias integradas regionalmente en bloques funcionales de comercio (UE) o economias de alto poder transaccional (por ej. USA y Japón), construyen estrategias comerciales calificables de "neo-proteccionistas" al margen de las negociaciones y acuerdos del GATT-OMC; estas implican la utilización de barreras no arancelarias. RVE, Subsidiación, barreras tecnológicas y precios mínimos (tigger price mechanism). La justificación suele ser la existencia de "unfair trade", salarios bajos que se traducen en "dumping económico" y países cerrados por importaciones (Japón frente a USA).

10 Las críticas que se hacen a la industrialización, sustitutiva de importaciones en América Latina es su aplicación excedida a su fase de agotamiento, su aplicación desvinculada de la industrialización orientada a las exportaciones -EOI-, es decir con respecto a una política de estímulos a las exportaciones (tal como el sistema asiático "export-import link system"). El resultado fue una "fatiga de protección: que distorsionó los propósitos del modelo. Puede leerse en P. Bustelo (1992). 
lo de intercambios simultáneos: desembolsos-condicionalidades-esfuerzo de ajuste.

La lógica institucional o "performance criteria"11 de estas entidades conduce, entonces, inevitablemente, a la apertura y desregulación de relativa indiscriminación ${ }^{12}$.

Sin una estretegia interna de crecimiento económico y sin suficiente consenso político y social, rechazar la opción de la institucionalidad washingtoniana sería equivalente a su exclusión del orden económico. Las experiencias de proyectos nacionalistas a espaldas de dicha institucionalidad, tales como la experiencia cubana o china, entre otras, han verificado la presunción del fracaso. La comparación entre éstas con el "despegue" de las economias asiáticas o de la China "abierta" constituyen indicadores comprobatorios de la potencialidad de crecimiento asociada al comercio. Pero ¿son estas evidencias generalizables? P. Hirst y $G$. Thompson (1995) sostienen que los paises pobres se mueven en un espectro de adversidades: la autarquía les confina a la regresividad y la apertura, per se, les somete a la hostilidad de flujos comercia- les exclusores. Se requiere, de acuerdo a estos autores, de "nuevas estrategias y prioridades de las economías desarrolladas con respecto al tercer mundo", sin ello no habría beneficios en dejar atrás regímenes de clausura.

Aceptar la apertura "improvisada" $y$ condicionada exógenamente ha conducido, en términos generales, a exponer economías y sociedades desestructuradas, a fuerzas que sus propios Estados no pueden enfrentar. La inserción, no repensada, en un comercio aparentemente multilateralista sin protección estatal, en busca de una reciprocidad no garantizada ("quid pro quo") no parece generar los resultados que los partidarios del libre comercio aseguran ${ }^{13}$.

E. Ortíz (s.f.) sobre la ineficacia de la clausura comercial, afima "que no lleva a buenos resultados", y puntualiza que tampoco la apertura "es garantía de que estos últimos se conseguirán".

Aún cuando la liberación ha generado un crecimiento significativo del comercio internacional, en la producción mundial, han sido los países del "primer mundo" y algunas economías de industrialización reciente los receptores de los

11 La asistencia financiera del BM dirigida a sustentar los programas de reforma política e institucional, tiene como prioridad la reforma comercial. De hecho es "el área más sujeta a condicionalidades... casi el $80 \%$ de sus préstamos están condiclonados a lograr reformas en la política com. ercial, en segundo lugar las reformas del sector público y luego agricultura y sistema financiero. Puede leerse en D. Greenway y O. Morrisey (1993).

12 El "performance criteria" implica el cumplimiento de las condicionalidades a fin de asegurar no sólo la continuidad de los desembolsos sino tamblén la protección de "assets" del FMI. De alli su seguimiento a fin de detectar desviaciones. Para profundizar en esta materia léase M. Guitián (1995).

13 El BM y el FMI presentan al libre comercio como la mejor política (first best police). Sin embargo el costo de las primeras experiencias de apertura indiscriminada, permitieron la introducción de modificaciones en la gradualidad de la apertura y un cierto margen de proteccionismo. Puede leerse en D.Greenway y O. Morrisey. 
beneficios del mismo, y se estima que lo seguirán siendo ${ }^{14}$. Esta asimetría entraña un elemento de perturbación en el orden mundial que debilita, en el mediano plazo, la misma estabilidad política y social de las economías hoy exitosas; un elemento expresivo de ello lo constituyen los flujos migratorios "indeseables" desde los países no desarrollados; estos se han instituido en factores desorganizativos no sólo, de los mercados de trabajo de dichas economías, también de la gobernabilidad y la legitimidad social ${ }^{15}$.

La permanencia de la inequidad es, entonces, un "issue" de discusión inaplazable, también, para los países desarrollados involucrados en un juego de "suma cero". Existen elementos referenciales de concienciación institucional sobre la necesidad de reducción de los "gaps", redefiniendo los programas del Washington Consensus $y$, sosteniendo las políticas de ayuda (Ayuda Pública al Desarrollo). La UNCTAD por ejemplo, ha indicado al FMI la letalidad de programas de estabilización "destructivos de la prosperidad nacional o internacional" (Opschoor y Jongma, 1996). Esto ha incidido en la revisión de las condicionalidades, del antiactivismo estatal y de los programas de compensación. Por otra parte, desde organismos regionales (CEPAL) se aboga por la inclusión de "cláusulas de desarro110 " en las negociaciones (Agosin y Tussie, 1993).

\section{La legitimación de la intervención pública}

Habiéndose establecido que: a) la globalización determina, por lo menos, una alteración de la noción de EstadoNación, b) se asiste a una reducción de la capacidad de gerencia macroeconómica, agudizada en paises vulnerables con precaria capacidad agregativa y con Estados con cesión de soberanía, la liberalización en economias inmaduras, sin estrategia ni cláusulas de protección, no se asocia a crecimiento ni bienestar social; de alli que, en la literatura económica, encontramos una revisión de lecciones aprendidas e institucionalizadas las cuales nos dicen que la liberación garantiza el crecimiento, y que el "proteccionismo" estatal provoca desviaciones ineficientes con respecto a sus ventajas comparativas (estáticas). De acuerdo a tales lecciones los modelos de crecimiento han de suponer la liberalización incondicionada

14 De acuerdo a cálculos de la OCDE la liberación del comercio mundial originaría, hasta el año 2002, un incremento de la producción económica mundial de 274.000 millones de dólares; concentrando el "primer mundo" (UE/EFTA, Japón y EEUU) el $66 \%$ de este crecimiento. El "gap" tecnológico en términos de producción de alta tecnología mantendrá, también el mismo sesgo, concentrando éstos (sin incluir EIRs) el 63\% de la misma. Puede leerse en Rexrodt, G. (1993)

15 El mayor problema al que se enfrentan las economías de la UE es la alta tasa de desempleo (10\%: 17 millones de desocupados), sin posibilidad de resolución. La re-estructuración productiva, la sobre-oferta laboral de alta calificación asociada a la anexión de los países del este y las corrientes inmigratorias no encontrará, fácilmente una redistribución laboral en el espacio de la Unión. Es ésta una fuerza generadora de tendencias políticas radicales, tales como la etnofobia y los ultranacionalismos. Puede leerse en Delors, J. (1994) 
y la abstención pública en las estrategias de desarrollo. Sin embargo, el registro económico de las EIR ha invalidado el precepto teórico que condiciona liberalización e inactivismo estatal; en la actualidad el debate teórico se centra, precisamente, en la elección entre dos alternativas estratégicas de crecimiento con orientación hacia exportaciones: la participación gubernamental en la gestión de tales programas (intervención selectiva) versus su inactividad (principio de neutralidad).

Examinemos los argumentos más recientes sobre tal asunto:

1) El Estado como encendido de la "locomotora".

J. Stiglitz (1996) asigna al Estado "la mayor responsabilidad en la promoción del crecimiento" de EIR. Su tesis es expuesta a través de metáforas explicativas de las funciones desarrollistas del Estado: a) la metáfora de la "locomotora" o "engine of growth", de acuerdo a la cual el "rol del gobierno es encender la máquina para estimular altas tasas de acumulación de capital"; b) La metáfora del catalizador, en la cual aparece el Estado como generador de reacciones; realizando inversiones en capital humano e infraestructura para incrementar los retornos privados de la inversión y por ende promover el crecimiento; c) La metáfora biológica, expresiva del estado como "adaptati- ve system" es decir como organismo "altamente adaptativo" a los cambios en el entorno comercial internacional. Estas condiciones le confieren al gobierno la capacidad de diseñar agendas intervencionistas que, en lugar de "reemplazar mercados les promuevan y les usen", así como reducir conductas rentistas (rentseeking behavior) que provoquen resistencias adaptativas.

$¿$ Cuáles han de ser los puntos focales de intervención? políticas industriales, promoción de cooperación (más no colusión con empresas) y competencia, impulsos hacia la equidad y ambiente para inversiones extranjeras ${ }^{16}$.

2) El Estado desarrollista o "Developmental State" como gestor de un "Momento de Desarrollo"

Adrian Leftwich (1994) utiliza esta designación ${ }^{17}$ para definir la forma estatal requerida para promover el desarrollo, en oposición a la propuesta del BM del "good governance". El segundo alude a la eficiencia administrativa con aparente neutralidad política, el primero refiere a un "fuerte, determinado y relativamente autónomo Estado, democrático o no".

A éste se atribuye el "crecimiento económico sostenido en los países de desarrollo tardío (sea el mercado amigable o no)". La estrategia gubernamental, de acuerdo con Leftwich ha sido la elaboración de políticas que tiendan a concen-

16 Stiglitz (1996) enfatiza sobre la estrategia conducida por los gobiernos de las EIR para erradicar la aversión inicial a las inversiones extranjeras (xenophobic aversions to foreign investment).

17 Leftwich (1994) asume el concepto de Chalmer Johnson, venciendo la estigmatización que sobre este concepto se inicia en los ochenta. 
trar en el Estado, tanto las "competencias públicas como administrativas para establecer un momento de desarrollo en un ambiente internacional competitivamente hostil" ¿Cuáles son los atributos asociados a esta forma estatal?. La existencia de una élite gubernamental relativamente autónoma y poco corrupta (Ministerio de Industria y Comercio japonés), la capacidad de construcción de un sentido de unidad en torno a determinados objetivos de desarrollo (integración gobierno-empresarios) y de seguridad nacional.

3) El Estado como impulsor de "Leapfrogins"

Desde la vertiente teórica de los paradigmas Tecno-Económico y neoshumpeteriano, Lo Dic (1995) sostiene que "la mayoria de los paises en desarroIlo no son suficientemente aptos para alcanzar las <etapas del desarrollo> prometidas por el BM"; de alli que sea necesaria una estrategia estatal de industrialización basada en "leapfrogins" o "industrias de desarrollo que generan dinámjcas no concordantes con las ventajas comparativas dadas". La clave de esta propuesta es creer que el Estado debe, explícitamente, promover las desviaciones (diferenciaciones) apropiadas al escenario comercial existente.

Un argumento esgrimido por el autor es que las economías del sudeste asiático no han sido menos distorsionadas que las economías sustitutivas de importaciones de América Latina, pero el éxito de las primeras descansa en "la existencia de una forma apropiada de distorsión". La promoción de "Leapfrogins" por parte del estado, haria posible, entonces, "romper el círculo vicioso de espe- cialización en productos de baja cualificación y tecnología, junto a bajos niveles de ingreso". Las inversiones estatales en infraestructura, educación, y tecnología provocarian los "saltos" característicos de las "leapfrogins" e iniciaria con éstas, los conocidos "círculos virtuosos" del crecimiento.

\section{4) El Estado como promotor y orde- nador de "clusters" \\ Los "clusters" (Porter, 1990), cons-} tituyen "sites" o localizaciones de interacción empresarial e institucional que dan lugar a "sistemas nacionales de innovación" y a una "competencia sistemática y dinámica". ¿Cuál es el espacio gubernamental en este modelo de competitividad? De acuerdo a Porter, el desenvolvimiento económico registra tres secuencias hasta alcanzar ventajas competitivas sustentables: etapa de "factores básicos" (factor driven), caracterizada por el afianzamiento en la dotación natural de recursos, etapa de "factores avanzados" (advanced factors o investment driven), centrada en inversiones infraestructurales y tecnológicas para afianzar exportaciones y por último, el "timing" con sesgo innovador (innovation driven) o de "factores especializados". Hurtienne y Messner (1994), utilizando la teorización anterior, sostiene que en las dos primeras fases debe existir una activa intervención estatal.

De acuerdo a estos autores, "la mayoría de los países en vías de desarrollo atraviesa por una secuencia de desarrollo con "sesgo de factor" en la que las empresas "son muy sensibles a las fluctuaciones" en el mercado mundial. Mientras que en la segunda fase demanda gran- 
des inversiones infraestructurales no financiables por los grupos empresariales (start-up costs). En estas dos secuencias el Estado debe asumir "un papel rector", aplicando un instrumental de política económica de protección y consolidación: "aranceles tipo < infrant industry>, política crediticia selectiva e inversiones públicas masivas en educación e infraestructura básica" ${ }^{18}$. En la tercera secuencia, su función no será la de "promotor directo" sino de consolidante de los "clusters", creando el entorno institucional propicio y ordenando la competencia a través de "formas blandas de intervención".

\section{5) El Estado como "supervisor" del cre- cimiento}

M. Bruno (1994) de relativa cercanía analítica a la institucionalidad washingtoniana revela sin embargo, que el diferencial de éxito logrado por las EIR es adjudicable a "mejores poltticas macroeconómicas", específicamente a las que responden a los shocks externos. De acuerdo a este autor, el Estado debe monitoriar la exposición de los PND a la liberación, sugiriendo "una fuerte supervisión gubernamental". Aparece también en su tesis el argumento de la intervención selectiva focalizada en áreas delimitadas, aunque de fuerte exigencia de activismo estatal: inversión en intraestructura, cobertura de costos de arranque (start-up costs), programas sociales compensatorios, reforma institucional y credibilidad estatal.
6) El Estado como redistribuidor

E. Hobsbawm (1996) propone, ante la incertidumbre asociada a la erosión de los símbolos regulatorios, la necesidad, más acuciante que nunca, de preservar al Estado social; especificamente su función de redistribución. La globalización ha relativizado el tamaño de los Estados y debilitado su soberania; afirma por tanto que ello se refleja en el "hecho que los Estados se encuentran disminuidos para enfrentarse a la escala global, transnacional y ciertamente supranacional". Los efectos se expresan en un acrecentamiento de las desigualdades continentales, nacionales e intranacionales que no encuentran respuestas en las dos opciones de "orden" externas: el "anarquismo ultraliberalizador" $y$ el "small is beautiful". En ambos se desatan fuerzas exclusoras que marginalizan a la mayoría de la población mundial. De all la importancia de la preservación del Estado nacional y de su función redistributiva.

Al respecto Hobsbawm afirma que la función redistributiva, ha cobrado más importancia "ante las tendencias del desarrollo económico. La riqueza de las naciones desarrolladas continúa multiplicándose, pero esta riqueza creciente parece generada por una proporción menor de su población". Por tanto, a menos que surjan otros "mecanismos no mercantiles de redistribución, la provisión de seguridad social se construye alrededor de la autoridad pública".

18 Hurtienne y Messner (1994) definen al estado necesario en estas dos secuencias como "estado fuerte, de corte desarrollista". 
Puede concluirse, entonces, en que a pesar del éxito instrumental ${ }^{19} \mathrm{del}$ modelo "washingtoniano" de estrategia de crecimiento para los países no desarrollados (régimen de neutralidad), bajo el supuesto del "New Ideal", se ha forjado una corriente de oposición teórica que argumenta el re-establecimiento de la actuación estatal en la economía. La evidencia de soporte enunciada por los analistas presentados, la constituye el registro económico de las economias del sudeste asiático.

El desenvolvimiento de las mismas (Hong-Kong, Indonesia, Japón, Corea del Sur, Malasia, Singapur, Taiwan y Tailandia) se ha asociado, sin excepción a intervensiones estatales selectivas, flexibles pero también autónomas y férreas.

Es el "milagro asiático", precisamente, una de las referencias que permite creer en la posibilidad de insertarse en una trayectoria de crecimiento en un escenario de profundas asimetrías y ante fuerzas trans-territoriales que exceden la capacidad protectora de los Estados nacionales. El otro icono de posibilidades esta representado por los movimientos integrativos (bloques) que permiten la re-captura de la gobernabilidad y contestabilidad en un entorno que dista del enfoque de "amigabilidad del mercado" publicitado por el BM. Sin estos referentes, las tendencias exclusoras de la globalización harian predecible la marginalización secular de la mayoría de las economías inmaduras.

\section{Referencias bibliográficas}

Agosín, M. y Tussie, D. 1993. "Globalización, Regionalización y nuevos lemas de la política de comercio exterior para el desarrollo", El trimestre Económico Lx 559-599.

Bendesky, L. (1994). "La Economia regional en la era de la globalización", Comercio Exterior 44:932-949.

Bruno, M. (1994), "Development Issues in a Changing World: New Lessons, Old Debates, Open Question's. DeveIopment Economics: 9-19.

Bustelo, P. (1992). "La industrialización en América Latina y Asia Oriental: un análisis comparado", Comercio Exterior. $421111-1119$.

Delors, J. (1994). "Una entrevista a Jacques Delors", Deeutschland.

Greenway, D. y Morrisey, O. (1993). "Structural adjustment and Liberalisation in developing Countries: What lessons have we learned?, Kyklos 46 241262.

Guitian, M. (1995) "Conditionality: Past, Present, Future", JMF Staff Papers 42 792-835.

Hirst, P. y G. Thompson (1992) "The problem of globalization; international economic relation, national economic management and formation of trading blocs', Economy and Society 21 357-396.

(1995) "Globalization and future of the nation state" Economy and Society 24 408-442.

Hobsbawm, E.J. (1996) "The future of the State", Development and Change 27 267-278.

19 El exito instrumental de los programas de la institucionalidad washingtoniana es, en este articulo, entendido en términos de su extensiva aplicación. 
Hurtienne, T. y Messner, D. (1994). Camblo de Rumbo en el Cono Sur: crisis y oportunidades,Caracas: Editorial Nueva Sociedad.

Krugman, P. y Obstfeld, M. (1994) Economía Internacional, Madrid: McGraw-Hill.

Leftwich A. (1994), "Governance, the State and the Politics of Development" Development and Change 25;363-386.

Lo Dic (1995). "Techno-economic paradigm versus the market: on the recent theories of late industrialization", Economy and Society 24 443-470.

Markusen, J. (1995). "The boundaries of multinational Enterprises and the theory of international Trade". The Journal of Economic Perspectives 9(2) 169 . 190.

Ohmae, K. (1991). "El Mundo sin fronteras", México: McGraw-Hill.
Opschoor, J.B. y Jongma J.M. (1996). "Policy Options", Environment and Development Economics (3): 183-202.

Ortiz R., Eduardo (199__). "La politica Comercial y la transformación Productiva", Revista Venezolana de Economía y Clencias Sociales 2: 32.56 . (1996) Notas de clase en el seminario sobre Comercio Internacional en la F.C. E.S. de LUZ, Post-Grado de Macroeconomía y Política Económica, Junio de 1996.

Rains, G. (1995). "Another Look At The Asian Miracle", The World Bank Economy Review 9: 509-534.

Rexrod T.G. (1993). "Sobre el feliz término de las difíciles negociaciones del GATT y sus efectivos", Deutschland.

Sagasti, F. y Arévalo G. (1992), "América Latina en el nuevo orden mundial tracturado: perspectiva y estrategias" Comercio Exterior 42: 1102-1110. 